\title{
A Gradient Based Algorithm for PAPR Reduction of OFDM using Tone Reservation Technique
}

\author{
S. Janaaththanan, C. Kasparis, and Barry G. Evans
}

\begin{abstract}
In this paper, we propose a low complexity gradient based approach for enabling the Tone Reservation (TR) technique to reduce the Peak-to-Average Power Ratio (PAPR) of Orthogonal Frequency Division Multiplexing (OFDM) signals. The performance of the proposed algorithm is evaluated for different pilot location in the frequency domain, and also in combination with the Discrete Fourier Transform (DFT) spreading technique proposed in [6]; in order to further reduce the PAPR. Simulation results show that the new technique achieves significant PAPR reductions, which are further enhanced when it is combined with DFT spreading. The simulation results also show that the performance of the technique is dependent on the pilot positions. In addition, further investigation was performed where the reduction tones are constrained, equal to the average power mask for the data tones, by a simple projection rule in the frequency domain both for the TR scheme and for the combined scheme. Simulation results show that the contiguous pilot arrangement provides better PAPR reduction performance in both cases, when the peak-cancellation signal is constrained in the frequency domain.
\end{abstract}

Index Terms-OFDM, PAPR, Tone Reservation, DFT, spreading, DSI( Dummy Sequence Insertion).

\section{INTRODUCTION}

$\mathrm{T}$ HE performances of high data rate systems are significantly limited by the multipath interference that occurs in the radio channel environment. As an attractive technique in mitigating the multipath interference, Orthogonal Frequency Division Multiplexing (OFDM) has been widely applied in various high data rate wireless Local Area Networks (LAN) and broadcasting systems [1],[2]. Furthermore, it is also being considered in 3GPP Long Term Evolution (LTE) as an attractive multiple access technique in the downlink [3].

Despite its competitive attributes, OFDM signals are characterized by very high Peak-to-Average Power Ratio (PAPR) levels and this can both degrade the link performance and also cause significant out-of-band interference products that make it harder for the operator to comply with stringent spectral masks. The simplest solution to this problem is to operate the high power amplifier in the linear region by allowing a large enough amplifier back-off. However, this approach degrades the power efficiency of the system and often leads to unacceptable cost-efficiency conditions in the overall system [2].

Based on this background, reducing the PAPR of OFDM signals is increasingly being considered to be very important in maintaining the cost-effectivess advantages of OFDM in practical systems, especially as new systems are being specified with large number of sub-carriers. Up to date, there have been proposed various PAPR reduction techniques such as Clipping method, Block Coding method, Selective Mapping (SLM) method, Partial Transmit Sequence (PTS) method, and Tone Reservation (TR) method [2]. According to [2], Clipping method does not offer an attractive solution, because it leads to out-of-band distortion. Furthermore, the block coding method is not popular since it works on the trade-off between the PAPR performance and bandwidth efficiency. On the other hand, the SLM and PTS methods reduce the PAPR by controlling the phase of the data sub-carriers, which provides an effective solution. However they are computationally expensive and also require transmitting continuous side information to the receiver, which degrades the capacity of the system. The overall BER performance may also be degraded if there are errors in the side information [2]. In more detail, the TR technique was proposed by Tellado [4], whereby a small number of sub-carriers are reserved to create a cancellation signal in the time domain, which can cancel the high peaks in the information carrying signals at the transmitter. The cancellation signals can be easily discarded at the receiver after the FFT processing. In this way, the technique can reduce PAPR of the OFDM signal without introducing additional distortions to the information content and does not require side information to be sent to the receiver. However, the TR algorithms available, so far, in the literature leads to high computational complexity when the spectral constraints are to be incorporated, for example the active-set approach proposed in [5].

In this paper, we propose a gradient-based TR algorithm for creating the peak-cancelling signal in the time domain by utilizing the reserved sub-carriers. Furthermore, the proposed algorithm solves in an efficient manner the problem in imposing the spectral constraints on the peak-cancelling signal; the spectral constraint is employed directly in the frequency domain and thereby, leads to a tremendous reduction in the complexity. The proposed algorithm is then combined with the DFT spreading technique proposed in [6], in order to further reduce the PAPR. Investigating the combination of the two PAPR reduction techniques is 
particularly relevant for the Single Carrier-Frequency Domain Multiple Access (SC-FDMA) which inherently applies the DFT spreading, and is considered as strong candidate multiple access technique for the return link in LTE- UMTS [3].

The remainder of this paper is organized as follows: Section II introduces the system model, a brief derivation of the proposed gradient-based algorithm together with a brief introduction about OFDM transmitter and the definition of PAPR. In Section III, we present the simulation results for both for the proposed algorithm and also for the combined scheme. Furthermore, investigation was extended in both cases to incorporate the frequency domain constraints, equal to the average power mask for the data tones, on the peakcancelling signals and the corresponding simulation results are reported. Finally, the conclusion is given in Section IV.

\section{SYSTEM MODEL}

In this section, we first briefly review the basic of the OFDM transmitter and the PAPR definition. Moreover, a brief step-by-step derivation of the proposed gradient based algorithm is also exposed.

\section{A. $O F D M$ and PAPR}

The OFDM signal is the sum of many orthogonally overlapped sub-channels of equal bandwidth. In order to realize these spectrally overlapping sub-channels, the Inverse Fast Fourier Transform (IFFT) is employed at the OFDM transmitter. The base-band samples for OFDM symbol, with $N$ sub-carriers, at the output of IFFT (Inverse Fast Fourier Transform) output is given by [1], [2]:

$$
x_{n}=\frac{1}{\sqrt{N}} \sum_{0}^{N-1} X_{k} e^{j 2 \pi n k / N}
$$

Where $n=0,1, \ldots \ldots, N-1$ and $X_{k}$ denotes the data symbol at the $\mathrm{k}^{\text {th }}$ sub-carrier.

The Peak-to-Average Power Ratio (PAPR) of a signal is defined as [1]:

$$
P A P R=\frac{\max _{r \in n}\left|x_{r}\right|^{2}}{E\left[\left|x_{n}\right|^{2}\right]}
$$

Where $E\left[\left|x_{n}\right|^{2}\right]$ is the expectation value, represents the average power of the signal.

Since Nyquist-rate samples might not reveal the peaks of the continuous-time signal, it is desirable to show PAPR performance on over-sampled discrete-time signals [2]. It is typical to use an over-sampling rate of $L \geq 4$ so that the PAPR before the digital-to-analog (D/A) conversion can accurately describe the PAPR after the D/A conversion [4].This oversampling is done by simply performing the IFFT to the frequency domain symbol vector, $\mathbf{X}$, formed by concatenating $N(L-1)$ zeros to the original frequency domain vector [2]:

$\mathbf{X}=\left[X_{0}, X_{1}, \ldots \ldots, X_{N / 2-1}, 0, \ldots \ldots . .0, X_{N / 2}, \ldots \ldots, X_{N-1}\right]$

And the resulting time domain samples are:

$$
\mathbf{X}=\mathbf{Q X}
$$

Where $\mathbf{Q}=\left[\boldsymbol{Q}_{\boldsymbol{l m}}\right]_{\boldsymbol{N} \mathbf{L} \times \boldsymbol{N} \boldsymbol{L}}$ is the IFFT matrix with $\boldsymbol{Q}_{\boldsymbol{l m}}=$ $\frac{1}{\sqrt{N L}} \boldsymbol{e}^{j 2 \pi(l-1)(m-1)}, l=1,2, \ldots, N L$ and $m=1,2, \ldots \ldots, N L$.

\section{B. Proposed Low Complexity Gradient Based Algorithm for} TR technique

If we assume that $V$ tones are assigned to $V$ sub-carriers with indexes $\left\{l_{1}, l_{2}, \ldots ., l_{V}\right\}$ in the OFDM transmitter (before over-sampling), equation (3) can be decomposed into (4):

$$
\mathbf{x}=\mathbf{U} \boldsymbol{\rho}+\mathbf{W Y}
$$

Where $\boldsymbol{\rho}$ is a vector representing the tones to be used for PAPR reduction, $\mathbf{U}$ is the matrix with corresponding columns of $\mathbf{Q}$ and $\mathbf{Y}$ is the vector with information symbols and $\mathbf{W}$ is the matrix with corresponding columns of $\mathbf{Q}$. This can be further simplified into:

$$
\mathbf{x}=\mathbf{U} \boldsymbol{\rho}+\mathbf{y}, \quad \text { where } \mathbf{y}=\mathbf{W Y}
$$

As common to all PAPR reduction schemes, our task now is to reduce the cost function:

$$
\mathrm{J}_{\infty}=\|\mathbf{x}\|_{\infty}=\|\mathbf{U} \boldsymbol{\rho}+\mathbf{y}\|_{\infty}
$$

This is an infinity-norm minimization problem. This infinite-norm function in (6) is a non-smooth function and finding the exact solution is a tedious task. Therefore, we take an approximated approach based on the p-norm approximation for infinite-norm, given by [8]:

$$
\mathrm{J}_{\mathrm{p}}=\lim _{\mathrm{p} \rightarrow \infty}\|\mathbf{x}\|_{\mathrm{p}}
$$

According to [7], this p-norm function can be shown as a convex function for any value of $\mathrm{p}$. Therefore, the task now is to find the gradient of this p-norm function and then deduce the gradient of the infinite-norm as $\mathrm{p}$ tends to infinity.

$$
\frac{\partial \mathrm{J}_{\mathrm{p}}}{\partial \rho}=\left(\mathrm{J}_{\mathrm{p}}\right)^{1-\mathrm{p}} \sum_{\mathrm{r}=1}^{\mathrm{LV}}\left|\mathrm{x}_{\mathrm{r}}\right|^{\mathrm{p}} \mathrm{U}_{\mathrm{r},:} \mathrm{x}_{\mathrm{r}}
$$

When $\mathrm{p} \rightarrow \infty$, equation (8) becomes [8]:

$$
\frac{\partial \mathrm{J}_{\infty}}{\partial \boldsymbol{\rho}} \approx\left|x_{b}\right|^{-1} \mathbf{U}_{b,:} x_{b}
$$

Where the subscript $b$ represents the index of the maximum magnitude of the output sample.

Using this gradient, we formulate a simple gradient based algorithm with the step size parameters as:

$$
\mu_{i t e r}=\frac{\alpha}{i t e r}
$$

Where $\alpha=0.3$, found using the trial and error method and iter denotes the number iterations. A step size selection similar to (10) was considered in [7].

The gradient based equation for updating the reserved pilots becomes [9]:

$$
\boldsymbol{\rho}^{\mathrm{i}}=\boldsymbol{\rho}^{\mathrm{i}-1}-\mu^{i}\left|x_{b}\right|^{-1} \mathbf{U}_{b,:} x_{b}
$$

Since this update rule is performed in the frequency domain, this algorithm can simply incorporate the necessary spectral constraint, by simply limiting the power amplitude or power of the pilot tones. As a result, after the update given by 
(11), we apply the following projection rule to each component of $\boldsymbol{\rho}$ :

$$
\mathbf{P}\left(\rho_{\mathrm{k}}\right)= \begin{cases}\rho_{\mathrm{k}} & \left|\rho_{\mathrm{k}}\right| \leq \beta_{\mathrm{k}} \\ \frac{\beta_{\mathrm{k}}}{\left|\rho_{\mathrm{k}}\right|} \rho_{\mathrm{k}} & \left|\rho_{\mathrm{k}}\right|>\beta_{\mathrm{k}}\end{cases}
$$

Where $\beta_{\mathrm{k}}$ 's are the magnitude constraint for the $\mathrm{k}^{\text {th }}$ component of $\boldsymbol{\rho}$. This projection operation is consistent with the Gradient Projection method [11] for minimizing functions over convex sets.

\section{SIMULATION RESULTS}

TABLE I

SIMULATION PARAMETERS

\begin{tabular}{ll}
\hline \hline Parameter & \multicolumn{1}{c}{ Value } \\
\hline Num. of Sub-carriers & 256 \\
Number of Pilots & 8 \\
Modulation & QPSK \\
DFT spreading & 248 \\
Performance Metric & CCDF \\
Num. of Iterations & 50 \\
Pilot Positions & \\
Position 1 & Equi-spaced starting from first location. \\
Position 2 & Suffix with the data block \\
Position 3 & Randomly found best Locations \\
\hline
\end{tabular}

The performance of the proposed algorithm was evaluated by means of computer simulations. All the simulations were carried out for $10^{5}$ OFDM blocks. The corresponding simulation parameters are given in Table 1. All performances were evaluated based on the Complementary Cumulative Distribution Function (CCDF), which will give the probability that the $P A P R$ is higher than a value, $P A P R_{0}$.

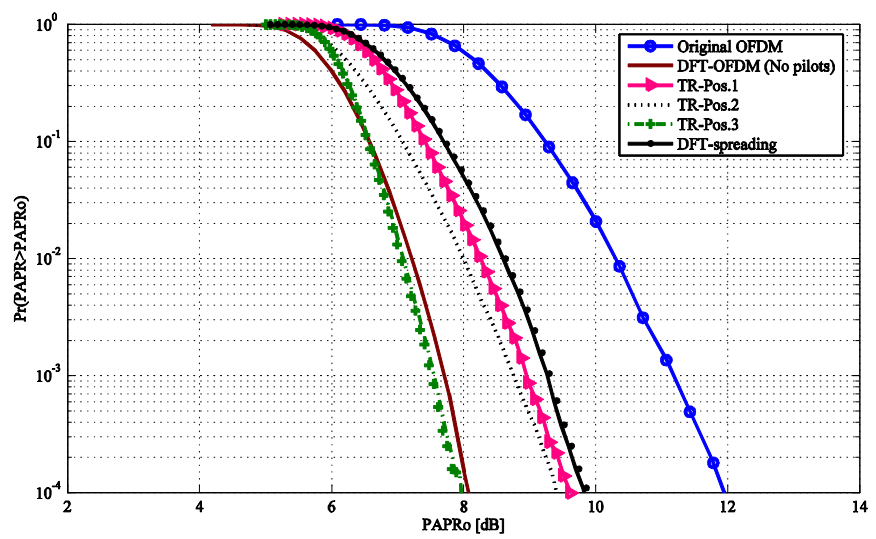

Fig. 1. PAPR Reduction Performance of OFDM for different pilot locations- TR technique only.

Figure 1 shows the simulations results with the proposed algorithm for different pilot locations. It is observed that the TR technique can achieve better results that the DFT spreading technique proposed in [6]. However, its performance appears to be dependent on the pilot arrangement.
Notably, TR technique needs to sacrifice a small fraction of the bandwidth for PAPR reduction purpose and thus, leads to negligible data rate loss. However, for a system which does not need to cope with bandwidth scarification, the proposed algorithm can be, without further modification, easily incorporated to the technique proposed in [10]. In which, the designed peak-cancelling signal is employed in the nullsubcarriers in the OFDM systems; therefore, it is called as Dummy Sequence Insertion (DSI) method. Figure 2 explains a typical example of the OFDM symbol and pilot arrangement used in such technique. In the DSI technique, unlike PTS and SLM method, the phases of the dummy sub-carriers are controlled to reduce the PAPR of the OFDM signal. Hence, the transmitter needs not to be communicated with the receiver for side information.

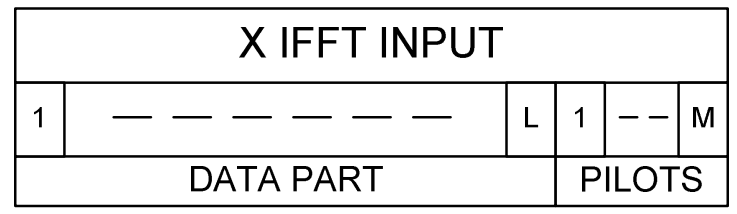

Fig. 2. A typical example for Dummy Sequence Insertion (DSI) technique with $\mathrm{L}$-data symbols and M-pilot symbols.

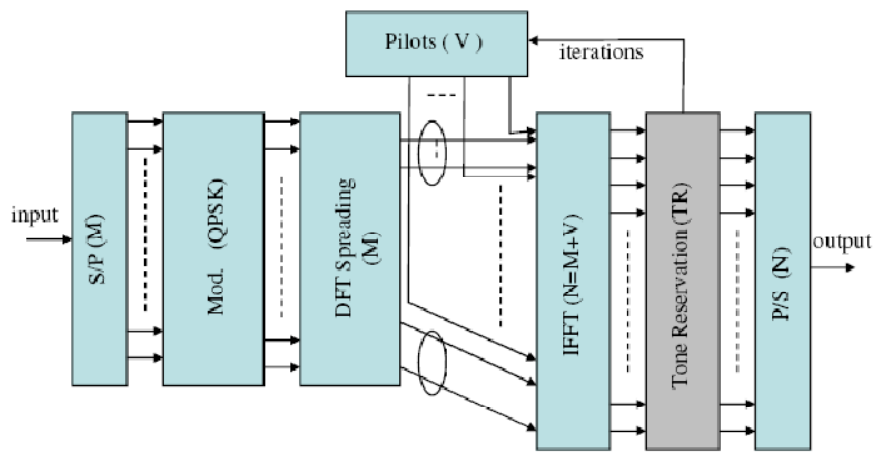

Fig.3. Block Diagram of the combined scheme: TR and DFT Spreading Techniques

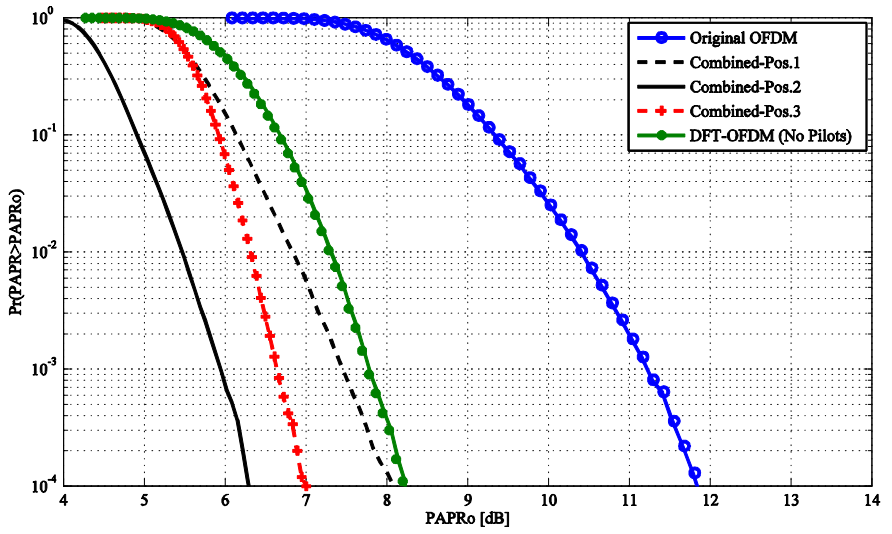

Fig. 4. PAPR Reduction Performance of OFDM for different pilot locations- Combined technique. 
Moreover, the TR method (or DSI method) can simply be combined with other PAPR reduction techniques to reduce the further PAPR in the OFDM signal. Figure 3 shows a possible combination of the TR method with the DFT spreading technique proposed in [6].

Figure 4 shows the performance of the combined TR and DFT spreading schemes for different pilot locations. The best performance was obtained when the pilots are suffixed to the data. However, similar performance can be asymptotically achieved with the pilot location chosen using trial and error method. In both cases, equally spaced pilot locations are not preferred over the other two pilot arrangements.

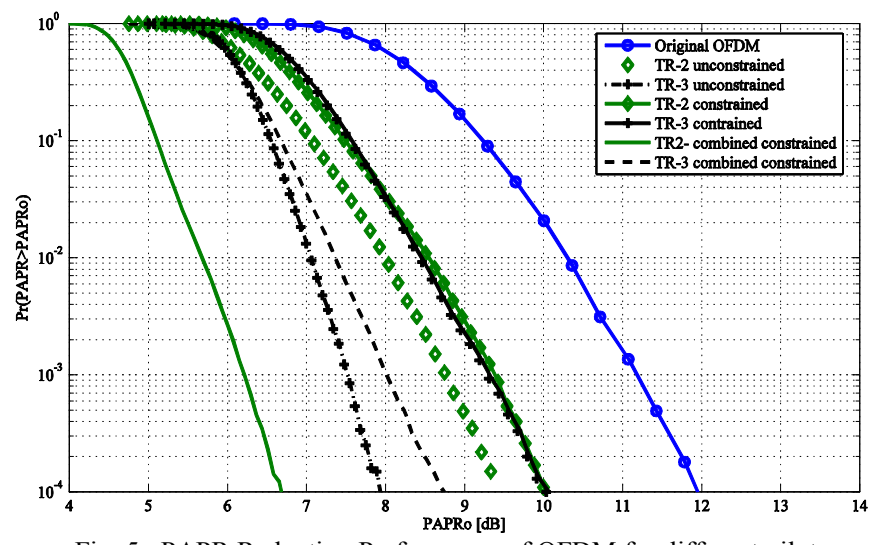

Fig. 5. PAPR Reduction Performance of OFDM for different pilot locations- constraints applied.

Figure 5 shows similar simulation results as Figure 1 and Figure 4, but using the restrictive spectral constraints, equal to the average power mask for the data tones, in the frequency domain as given by the projection rule in equation (12). It is observed that introducing spectral constraints into the proposed algorithm significantly affects the achievable PAPR reduction, except for the case when the pilot tones are suffixed with the data block similar to Figure 2; where only a negligible reduction in the achievable PAPR reduction. However, the performance is mostly affected when the pilots are located randomly according to the position 3 .

\section{CONCLUSION}

In this paper, we proposed a novel gradient based approach for PAPR reduction using Tone Reservation technique. We showed that proposed algorithm performs better than the DFT spreading technique proposed in [6]. However, the performance depends on the pilot locations.

We also showed the combined scheme (DFT spreading+TR) performance much better than the both techniques, providing double the performances achieved using the single technique.

From the extended investigation using the spectral constraints, we showed that introducing constraints into the proposed algorithm affects the achievable PAPR reduction in all the cases. However, there is only a negligible reduction on the achievable PAPR reduction when the pilots are suffixed with the data (position 2) in the combined schemes.
Since 3GPP has recently launched a study item called 3GPP LTE (Long Time Evolution). In which, SC-FDMA is considered as the uplink candidate which uses DFT spreading to reduce the PAPR. Therefore, this combined scheme is also appropriate in that type of systems.

\section{ACKNOWLEDGEMENTS}

The authors would like to acknowledge the European Satellite Network of Excellence (SatNEx2) for funding this work.

\section{REFERENCES}

[1] R. Van Nee and R. Prasad, OFDM for Wireless Multimedia Communications, Norwood, MA:Artech House, 2000.

[2] Seung Hee Han, and Jae Hong Lee, "An Overview of Peak-to-Average Power Ratio Reduction Technique for Multicarrier Transmission," IEEE Wireless Comm., vol.12, pp. 56-65, Apr. 2005.

[3] 3GPP TR 25.913 Requirements for Evolved UTRA (E-UTRA) and Evolved UTRAN (E-UTRAN, V.7.0).

[4] J. Tellado, Peak-to-Average Power Reduction for Multicarrier Modulation, Ph.D. Thesis, Stanford University, Sept. 1999.

[5] Brian S Krongold and Douglas L. Jounes, "An Active-Set Approach for OFDM PAR Reduction via Tone Reservation", IEEE Transactions on Signal processing., vol. 52, no 2, pp.495-509, February 2004.

[6] Heung-Gyoon Ryu, and Sang-Woo Kim," PAPR Reduction using DFT Transform of the OFDM Signal for the High-Speed Satellite Communication," 25th AIAA ICSSC'07, AIAA 2007-3299, Jun. 2007.

[7] S.Boyed and L.Vandenberghe," Convex Optimization", Cambridge University Press, 2004.

[8] Gonzalez, A.; Albiol, A.; Elliott, S.J., "Minimization of the maximum error signal in active control," Speech and Audio Processing, IEEE Transactions on Speech and Audio Process., vol.6, no.3, pp.268-281, May 1998

[9] Simon Haykin," Adaptive Filter Theory", $3^{\text {rd }}$ ed., Prentice-Hall, New Jersey, 1996.

[10] Heung-Gyoon Ryu, Jae-Eun Lee, and Jin-Soo Park,"Dummy Sequence Insertion (DSI) for PAPR Reduction in the OFDM communication system", Consumer Electronics. Letter, vol. 50, no. 1, Feb. 2004.

[11] E. S. Levitin and B. T. Polyak, "Constrained minimization methods", USSR computational Mathematics and Mathematical Physics, vol. 6, pp. 1-50, 1966. 